\title{
Linking Activation of Microglia and Peripheral Monocytic Cells to the Pathophysiology of Psychiatric Disorders
}

\author{
Yuta Takahashi ${ }^{1,2,3}$, Zhiqian Yu ${ }^{1,2,4}$, Mai Sakai ${ }^{1,2}$ and Hiroaki Tomita ${ }^{1,2,4 *}$ \\ ${ }^{1}$ Department of Disaster Psychiatry, International Research Institute of Disaster Science, Tohoku University, Sendai, Japan, \\ ${ }^{2}$ Department of Disaster Psychiatry, Graduate School of Medicine, Tohoku University, Sendai, Japan, ${ }^{3}$ Department \\ of Psychiatry, Graduate School of Medicine, Tohoku University, Sendai, Japan, ${ }^{4}$ Group of Mental Health Promotion, Tohoku \\ Medical Megabank Organization, Tohoku University, Sendai, Japan
}

\section{OPEN ACCESS}

Edited by:

Takahiro A. Kato,

Kyushu University, Japan

Reviewed by:

Andrew MacLean,

Tulane University, USA

Masahiro Ohgidani,

Kyushu University, Japan

*Correspondence:

Hiroaki Tomita

htomita@med.tohoku.ac.jp

Received: 08 March 2016 Accepted: 16 May 2016

Published: 03 June 2016

Citation:

Takahashi Y, Yu Z, Sakai M and Tomita H (2016) Linking Activation of Microglia and Peripheral Monocytic Cells to the Pathophysiology of

Psychiatric Disorders.

Front. Cell. Neurosci. 10:144.

doi: 10.3389/fncel.2016.00144
A wide variety of studies have identified microglial activation in psychiatric disorders, such as schizophrenia, bipolar disorder, and major depressive disorder. Relatively fewer, but robust, studies have detected activation of peripheral monocytic cells in psychiatric disorders. Considering the origin of microglia, as well as neuropsychoimmune interactions in the context of the pathophysiology of psychiatric disorders, it is reasonable to speculate that microglia interact with peripheral monocytic cells in relevance with the pathogenesis of psychiatric disorders; however, these interactions have drawn little attention. In this review, we summarize findings relevant to activation of microglia and monocytic cells in psychiatric disorders, discuss the potential association between these cell types and disease pathogenesis, and propose perspectives for future research on these processes.

Keywords: microglia, monocyte, mental disorder, psychoimmunology, peripheral biomarker, blood-brain barrier, schizophrenia, bipolar disorder

\section{INTRODUCTION}

A variety of postmortem brain studies and recent positron emission tomography (PET)-based studies have identified microglial activation in psychiatric disorders, such as schizophrenia (Bayer et al., 1999; Radewicz et al., 2000; van Berckel et al., 2008; Tang et al., 2012; Fillman et al., 2013), bipolar disorder (Haarman et al., 2014; Hercher et al., 2014), and major depressive disorder (Torres-Platas et al., 2014). Several studies have also indicated an association between alterations in monocytic features and psychiatric disorders (Rothermundt et al., 1998; Theodoropoulou et al., 2001; Padmos et al., 2008; Drexhage et al., 2011).

By contrast, peripheral monocytes can differentiate into macrophages and dendritic cells in peripheral tissues, both of which share similarities with microglia in their cellular morphology and functions, such as phagocytic activities, the expression of cell surface markers and cytokine production, and similar gene expression profiles (Schmitz et al., 2009; Beumer et al., 2012; Shechter and Schwartz, 2013; Prinz and Priller, 2014). Under pathological conditions in brain disorders, monocytes are recruited from peripheral blood into the brain, where they cooperate with

Abbreviations: CNS, central nervous system; LPS, lipopolysaccharide; NOD, non-obese diabetic. 
microglia in immune responses (Beumer et al., 2012; Shechter and Schwartz, 2013; Prinz and Priller, 2014). Considering the similarity and potency of the interactions between these two cell types, it is reasonable to speculate that correlations and interactions exist between the activation identified in microglia and peripheral monocytic cells in patients with psychiatric disorders, although these possibilities have drawn little attention. In this review, we summarize the accumulated findings regarding microglial and peripheral monocytic activation in psychiatric disorders and discuss the potential mechanisms linking microglial and monocytic activation with the pathogenesis of psychiatric disorders. Finally, we propose directions for future research on these potential associations.

\section{ACCUMULATED FINDINGS OF MICROGLIAL ACTIVATION IN PSYCHIATRIC DISORDERS}

Postmortem brain studies have suggested an association between psychiatric disorders and microglial activation (Bayer et al., 1999; Radewicz et al., 2000; Tang et al., 2012; Fillman et al., 2013; Hercher et al., 2014; TorresPlatas et al., 2014). Regarding the qualitative assessment of microglial morphology, Hercher et al. (2014) observed that activated microglial cells were increased in prefrontal white matter from patients with schizophrenia, but not that from patients with bipolar disorder. Torres-Platas et al. (2014) showed that primed (activated) microglia were significantly increased compared with ramified (resting) microglia in the anterior cingulate cortices obtained from patients who died of depressed suicides compared with healthy controls, whereas the total densities of ionized calcium-binding adapter molecule 1 (IBA1) positive microglia did not differ between the depressed suicide cases and controls. Bayer et al. (1999) and Radewicz et al. (2000) reported that the expression of Human Leukocyte Antigen-antigen D Related (HLADR), which reacts with activated microglia, was increased in the frontal cortices of patients with schizophrenia in immunostaining studies. Fillman et al. (2013) demonstrated that the interleukin-6 (IL-6), IL8, and IL1ß mRNA transcripts were over-expressed in the dorsolateral prefrontal cortex, and the density of major histocompatibility complex class II (MHC-II) receptor-positive microglia (i.e., antigen-presenting cells) was increased in the white matter of patients with schizophrenia. Tang et al. (2012) found positive correlations among several activated microglial markers in subjects with schizophrenia. They also showed that changes in the expression of genes that encode markers of activated microglia were associated with inflammatory markers in the arachidonic acid signaling pathway in patients with schizophrenia.

Recent PET-based evaluations of microglial activation may also be applicable to psychiatric patients (Veenman and Gavish, 2000, 2012; Papadopoulos et al., 2006; van Berckel et al., 2008; Doorduin et al., 2009; Takano et al., 2010; Haarman et al., 2014). PET radioligands, such as C-PK11195 and DAA1106, are selective for the $18 \mathrm{kDa}$ translocator protein/peripheral benzodiazepine receptor, which is highly expressed in activated microglia and is involved in multiple cellular processes, such as apoptosis, the regulation of cellular proliferation, immunomodulation and steroidogenesis (Veenman and Gavish, 2000, 2012; Papadopoulos et al., 2006). van Berckel et al.'s (2008) PET study showed a significant increase in microglial activation in patients with schizophrenia who had a disease onset within 5 calendar years compared with controls, although all patients were under treatment with atypical antipsychotics, and confounding effects of drug treatments remain unexcluded. Interestingly, Doorduin et al. (2009) in a PET study of patients who had recovered from psychosis, observed no significant microglial activation. Although Takano et al. (2010) found no significant difference between $\left[{ }^{11} \mathrm{C}\right] \mathrm{DAA} 1106$ binding in normal controls and patients with schizophrenia, the patients exhibited positive correlations between cortical $\left[{ }^{11} \mathrm{C}\right] \mathrm{DAA} 1106$ binding, positive symptom scores and duration of illness. Haarman et al. (2014) observed a significant increase in ${ }^{11} \mathrm{C}-\mathrm{R}-\mathrm{PK} 11195$ binding potential in the right hippocampus of patients with bipolar disorder type I compared with healthy controls.

\section{MICROGLIAL FUNCTION AND POTENTIAL MECHANISMS UNDERLYING THE PATHOGENESIS OF PSYCHIATRIC DISORDERS}

Microglia comprise $\sim 12 \%$ of cells in the central nervous system (CNS; Vaughan and Peters, 1974); these cells are not uniformly distributed (Schmitz et al., 2009). More microglia are located close to neurons in the gray matter, with the highest concentrations in the hippocampus, olfactory telencephalon, basal ganglia, and substantia nigra (Lawson et al., 1990).

Accumulating evidence from fate-mapping studies suggests that the origin of most microglia is not the bone marrow after birth but hematopoietic stem cells in the yolk sac in the early developmental stage (Lassmann et al., 1993; Ginhoux et al., 2010; Schulz et al., 2012; Kierdorf et al., 2013; Prinz and Priller, 2014). Novel transgenic approaches have shown clear differences in the cellular characteristics of microglia and macrophages in the brain (Goldmann et al., 2013; Parkhurst et al., 2013; Yona et al., 2013). In contrast to macrophages, microglia are long-lived and are not replaced by circulating peripheral monocytes under physiological conditions.

Microglia can be polarized into two subtypes: M1 and M2, responding to certain stimuli. For examples, lipopolysaccharide (LPS) or interferon- $\gamma$ induces polarization into M1, whereas IL-4 or IL-13 induces M2 phenotype (Orihuela et al., 2016). Microglia contain two subtypes: M1 and M2. The M1 subtype is characterized by the production of pro-inflammatory cytokines, such as IL-1 $\beta$, IL-6, IL-8 and tumor necrosis factor alpha (TNF- $\alpha$; Barger and Basile, 2001; Boche et al., 2013). The 
M1 phenotype is activated by damage-associated molecular patterns, such as ATP, S100 molecules, histones, and heat shock proteins (Lu et al., 2014; Wiersinga et al., 2014). By contrast, the M2 phenotype is characterized by the production of anti-inflammatory cytokines, such as IL-10, insulin-like growth factor 1 (IGF-1), transforming growth factor beta (TGF- $\beta$ ), and neurotrophic factors (Ekdahl, 2012; Boche et al., 2013; Hu et al., 2015). The M2 phenotype is activated by cytokines, such as IL-4, IL-13 and IL-25 (Boche et al., 2013; Maiorino et al., 2013).

Activated microglia retract their cellular processes and transform from a ramified state into an ameboid morphology, in which they respond to external stimuli induced by various pathological conditions, such as trauma, infection, or other damage to brain tissue (Marshall et al., 2013). Activated microglial functions include phagocytosis and the production and release of cytokines, reactive oxygen species and nitrogen species (Barger and Basile, 2001; Takaki et al., 2012; Réus et al., 2015). They express a profile of cell surface marker expression that is similar to that of other mononuclear phagocytes (specifically macrophages), such as cluster of differentiation 14 (CD14), MHC molecules and chemokine receptors (Rock et al., 2004). Activation of microglia under pathological conditions in the brain may exert neuroprotective effects by reducing protein aggregates; however, they may exert cytotoxic effects by secreting neurotoxic factors (Streit et al., 1999; Schmitz et al., 2009).

\section{PERIPHERAL MONOCYTIC ACTIVATION IN PSYCHIATRIC DISORDERS}

Several studies have suggested an association between monocyte activity and psychiatric disorders (Rothermundt et al., 1998; Theodoropoulou et al., 2001; Nikkilä et al., 2014). Some studies have shown that circulating peripheral blood monocytes are increased in patients with schizophrenia (Rothermundt et al., 1998; Theodoropoulou et al., 2001). In addition, in the cerebrospinal fluid of patients with schizophrenia, the numbers of monocytes and macrophages were increased during acute psychotic episodes (Nikkilä et al., 2014). In contrast to schizophrenia, the number and level of CD14-positive monocyte differentiation were not altered in patients with bipolar disorder compared with healthy controls (Padmos et al., 2008; Drexhage et al., 2011). Recently, Drexhage et al. (2010) conducted a series of gene expression profiling studies using monocytes from psychiatric patients $(27$ schizophrenia and 56 bipolar patients) and matched controls via a microarray analysis, followed by quantitative polymerase chain reaction (PCR) studies for validation (Padmos et al., 2008; Beumer et al., 2012). The authors identified two main subsets of strongly correlated genes: one subset was composed of pro-inflammatory cytokines; the other subset consisted mainly of adhesion/motility factors. The monocyte gene expression profiles of the majority of the patients with bipolar disorder showed dysregulation in both subsets, whereas the monocyte gene expression profiles of the majority of the schizophrenia patients showed dysregulation only in the subset of pro-inflammatory cytokines (Drexhage et al., 2010).

\section{SUBPOPULATIONS OF PERIPHERAL MONOCYTIC CELLS AND POTENTIAL PATHOGENIC MECHANISMS UNDERLYING THE INVOLVEMENT OF PERIPHERAL MONOCYTES IN PSYCHIATRIC DISORDERS}

Monocytes are precursors of tissue macrophages, osteoclasts, and antigen-presenting cells (Lawson et al., 1990; Schmitz and Grandl, 2007). Monocytes, which comprise $5-10 \%$ of peripheral blood leukocytes, are derived from myelomonocytic stem cells in bone marrow and then released into the circulation, where they have a half-life of up to 3 days in humans (Whitelaw, 1972; Fogg et al., 2006). The brain harbors several types of monocyte-derived cells (Prinz and Priller, 2014). Macrophages and blood-derived dendritic cells are both present in the outer boundaries of the brain, such as the choroid plexus, perivascular space, and meninges; however, the number of blood-derived dendritic cells is small (Prinz and Priller, 2014).

There are five subsets of monocytes; they can be distinguished by different surface markers (Schmitz et al., 1997; Stöhr et al., 1998; Rothe et al., 1999; Gordon and Taylor, 2005; Beumer et al., 2012). More than half of monocytes belong to subset 1 and are characterized by surface marker profiles with abundant CD14 and a lack of CD16 expression (Schmitz et al., 1997; Stöhr et al., 1998). Both subsets 2 and 3 have CD16 expression and comprise active phagocytic cells. The expression of CD14 is increased in subset 2 compared with subset 3 (Schmitz et al., 1997; Stöhr et al., 1998). Subset 4 is a precursor of dendritic cells with high expression of CD40 (Schmitz et al., 1997; Stöhr et al., 1998). Subset 5, the smallest subset, shares many surface markers with subset 1 ; however, it differs in the additional expression of CD56, a marker of immature monocytes (Schmitz et al., 1997; Stöhr et al., 1998). Transformation between subsets among peripheral monocytes can occur concomitant with the differentiation of microglia in the brain under certain pathogenic conditions related to psychiatric diseases, although these possibilities remain to be elucidated.

\section{POTENTIAL MECHANISMS LINKING ACTIVATION OF MICROGLIAL AND PERIPHERAL MONOCYTIC CELLS TO PSYCHIATRIC DISORDERS}

As previously described, many studies have investigated the association between microglia and psychiatric disorders or monocytes and psychiatric disorders. However, only a few studies have investigated the direct association or interaction between microglia and monocytes. Theoretically, there are several potential mechanisms underlying these interactions, as described below (Figure 1). 


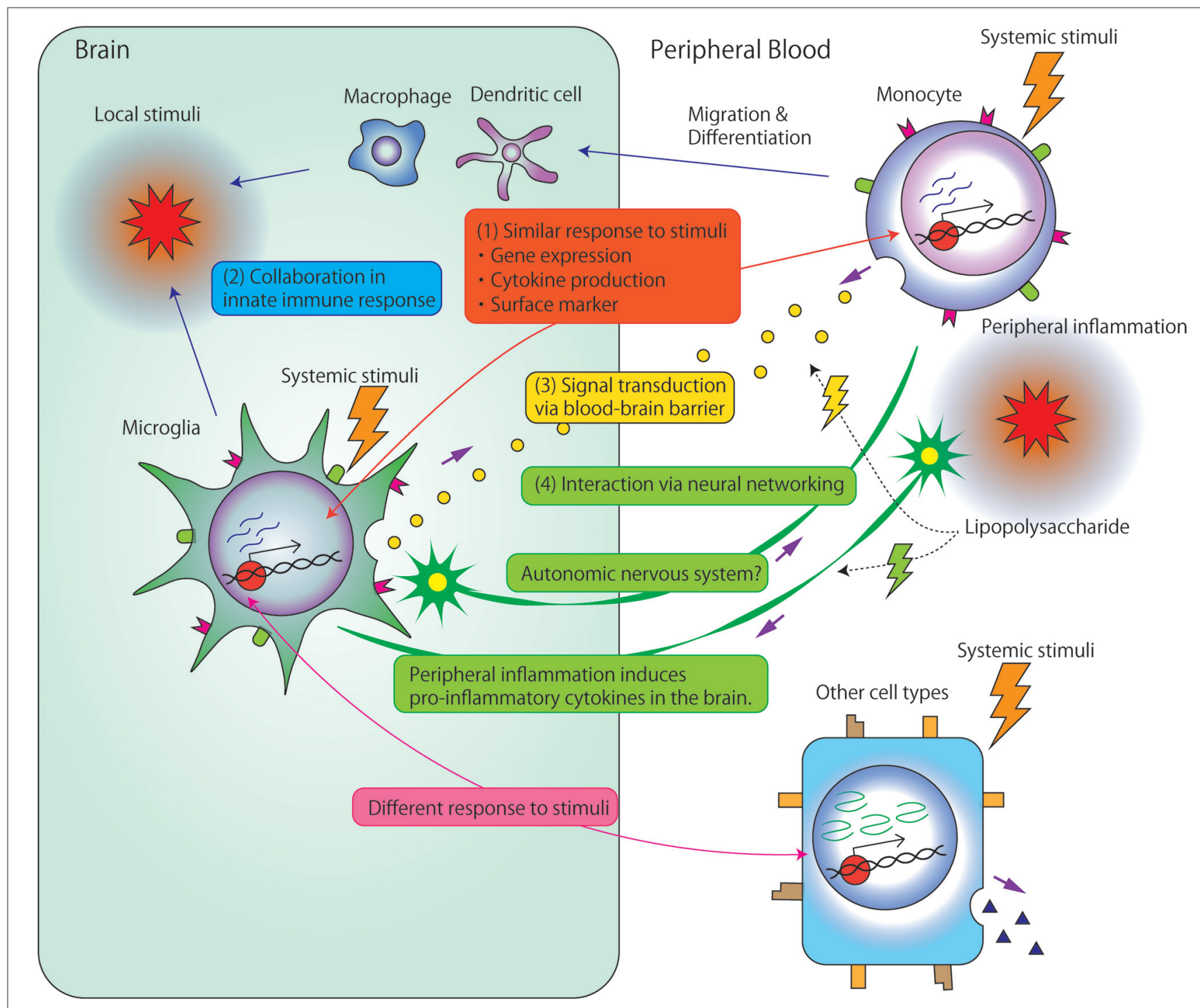

FIGURE 1 | Potential mechanisms underlying the associations and/or interactions between microglia and monocytes. (1) Microglia and monocytes exhibit similar responses to systemic stimuli. First, accumulated data have suggested strong associations between microglia and monocyte gene expression. Second, the two cell types show similar profiles for cytokine production, such as interleukin (IL) 1 $\beta$, IL-6, IL-8 or tumor necrosis factor (TNF)- $\alpha$. Third, both cell types express similar surface markers, such as cluster of differentiation 14 (CD14), major histocompatability complex (MHC) molecules, and chemokine receptors. (2) Local stimuli in the brain facilitate the recruitment of circulating monocyte-derived macrophage/dendritic cells into the central nervous system (CNS). Immigrant monocytic cells, which are short-lived, and localized in the outer boundaries of the brain compared with microglia (Prinz and Priller, 2014), may collaborate with microglia in the innate immune response. (3) Signals are transduced between microglia and circulating monocytes through the blood-brain barrier (BBB). Even relatively large molecules, such as cytokines, may comprise vehicles of signal transductions through circumventricular organs brain lymphatic pathways, BBB-relevant transporters/receptors, or abnormal permeability of BBB. (4) Microglia and peripheral monocytic cells may exert related biological reactions through neuronal signal transduction. Peripheral inflammation, induced by, for example, the peripheral administration of lipopolysaccharide (LPS), induces reactions in peripheral monocytic cells and also triggers the production of pro-inflammatory cytokines in the brain. Such reactions in the brain are thought to be induced by the transduction of neural excitability from peripheral nerves to the CNS because LPS rarely penetrates the BBB. Additionally, interactions between microglia and neuronal networks modulate myeloid cell proliferation through the autonomic nervous system.

\section{(1) Common Responses of Microglia and Peripheral Monocytic Cells to Endogenous or Exogenous Stimuli}

As mentioned in the introduction, microglia and monocytes have similar functions, such as phagocytosis and the release of pro-inflammatory cytokines, as well as similar expression of surface markers, such as CD14, MHC molecules, and chemokine receptors (Schmitz et al., 2009; Beumer et al., 2012; Shechter and Schwartz, 2013; Prinz and Priller, 2014). They may also share similar epigenetic marks, in addition to having the same genomic sequence. Although limited data directly indicate epigenetic similarities between microglia and peripheral monocytic cells, 
several previous studies have suggested similarities in the gene expression profiles of both microglia and peripheral monocytic cells. Schmitz et al. (2009) observed over-representation of genes associated with Alzheimer's disease among the expression profiles specific to microglia and monocytes (Thomas et al., 2006; Lutter et al., 2008). The authors compared the microglial gene expression profile with the microarray data of human blood monocytes and in vitro macrophage colony-stimulating factor (M-CSF) differentiated macrophages to assess the expression of genes associated with Alzheimer's disease. Of 379 Alzheimer's disease-related genes, 159 were expressed in microglia, 198 in monocytes and 206 in macrophages. Of these genes, the expression of 128 Alzheimer's disease-related genes were shared by microglia, monocytes and macrophages.

In addition, we compared the gene expression profiles of mouse microglia and monocytes in our preliminary microarray study prior to evaluating the effect of lithium treatment on microglia and peripheral monocytic cells (Yu et al., 2015). These findings also suggested a strong association between microglia and monocyte gene expression patterns. Among 45,281 transcripts on the microarray, 11,597 with average signal intensities greater than 100 in microglia or monocytes were reliably detectable. Of these transcripts, 7552 genes were identified in both cell types, including 2832 that were expressed only in microglia, and 1213 that were expressed only in monocytes. The correlation coefficients among the microglia samples were $0.94-0.98$, compared with $0.97-0.98$ for monocytes and $0.71-0.76$ between microglia and monocytes. By contrast, the alterations in the gene expression profiles following lithium treatment were quite different between the microglia and other types of monocytic cells. Yu et al. (2015) investigated the effects of lithium on two cell types: monocyte-derived dendritic cells treated with lithium and microglia separated from lithiumtreated mice. They compared the gene expression profiles of these cells with those of controls. They demonstrated that the common gene significantly induced by lithium in both monocyte-derived dendritic cells and microglia was only the third component of complement. Taken together, these findings indicate that microglia and monocytes have a similar gene expression pattern under normal conditions; however, changes in the pattern of expression profiles in response to stimuli are quite different.

\section{(2) Collaboration of Peripheral Monocytes with Microglia in the Innate Immune Response}

Damage to the CNS commonly results in the recruitment of circulating immune cells, including monocytes, which results in an innate immune response that consists of microglia and monocyte-derived macrophages/dendritic cells (Prinz and Priller, 2014). The differential roles of these myeloid cell populations in CNS disorders have only recently been acknowledged and were nicely illustrated in a recent review by Prinz and Priller (2014). In Alzheimer's disease, activated microglia have been associated with amyloid- $\beta$ induced neurotoxicity, and microglia were also damaged by amyloid species (Simard et al., 2006; Mildner et al., 2011; Prinz and Priller, 2014). Transplantation of wild-type bone marrow cells in transgenic mouse models of Alzheimer's disease causes the migration of bone marrow-derived phagocytes to the brain after CNS preconditioning. Consequently, the amyloid load in the brain is eliminated by bone marrow-derived phagocytes (Simard et al., 2006; Mildner et al., 2011; Prinz and Priller, 2014). Similarly, recovery from spinal cord injury in mice has been reported to depend more on infiltrating monocyte-derived macrophages than on resident microglia (Shechter et al., 2009).

\section{(3) Interactions Between Microglia and Peripheral Monocytic Cells Through the Blood-Brain Barrier}

Interactions between microglia and monocytes may also be regulated by cytokines. In a mouse model of amyotrophic lateral sclerosis, Butovsky et al. (2012) demonstrated that chemokine receptor-2 (CCR2) was over-expressed in splenic Ly6 $C^{\text {hi }}$ monocytes at disease onset, which was paralleled by upregulation of chemokine ligand-2 (CCL2) in CD39 ${ }^{+}$microglia. Additionally, CCR2 was not expressed in $\mathrm{CD}^{+} 9^{+}$microglia, and CCL2 was not expressed in Ly6C ${ }^{\text {hi }}$ monocytes during the pathological course of the disease. Therefore, the authors suggested that the expression of CCL2 and other chemokines on microglia caused the migration of Ly6 $\mathrm{C}^{\text {hi }}$ monocytes to the CNS.

Furthermore, both microglia and monocytes are activated by and release pro-inflammatory cytokines, such as IL-1 $\beta$, IL-6, IL-8 or TNF- $\alpha$ (Chan et al., 2007; Schmitz et al., 2009; Beumer et al., 2012). Studies have reported that these cytokines are increased in the blood of psychiatric patients (Naudin et al., 1997; Kim et al., 2000; Drexhage et al., 2008; Padmos et al., 2008; Song et al., 2009). Therefore, it is reasonable to suspect that these cytokine activation might underlie interactions between microglia and monocytes, although there has not been direct evidence to support the theory.

However, cytokines are relatively large molecules and rarely cross the blood-brain barrier (BBB). There are several known mechanisms and routes by which they can cross the barrier, such as alterations in the barrier's permeability (Esposito et al., 2001; Stamatovic et al., 2006), crossing though circumventricular organs (Anisman, 2009; Calderó et al., 2009; Banks and Erickson, 2010), or the use of specific transporters or receptors (Kastin et al., 1999; Chesnokova and Melmed, 2002; Banks and Erickson, 2010). Also, recent studies discovered functional lymphatic vessels lining the dural sinuses which can convey fluid, large molecules, and even immune cells from the brain and are connected to the deep cervical lymph nodes. Further researches into the CNS lymphatic system may facilitate understanding of interactions between microglia and peripheral monocytic cells (Iliff et al., 2015; Louveau et al., 2015).

\section{(4) Interactions Between Microglia and Peripheral Monocytic Cells Through Neuronal Networking}

Peripheral inflammation, which is caused, for example, by peripheral administration of LPS, induces peripheral reactions 
among immune cells, including monocytes. LPS also induces pro-inflammatory cytokines in the brain; however, LPS rarely crosses the BBB (Hayashi et al., 2015). This phenomenon may reflect that activation of inflammatory responses in the brain is induced by transduction of neural excitability from peripheral nerves to the CNS (Banks and Robinson, 2010), as well as LPS-induced dysfunction of vascular endothelial cells at the BBB (Banks et al., 2015). These findings suggest that mild peripheral inflammation, which contributes to the pathogenesis of psychiatric disease-related events, including fatigue, may induce microglial activation through neuronal networking.

Additionally, it is possible that interactions between microglia and neuronal networks modulate myeloid cell proliferation through the autonomic nervous system, although there is little evidence (Mignini et al., 2003; Spiegel et al., 2008). Peripheral monocyte activities may reflect microglial activities through these mechanisms.

\section{PERSPECTIVES ON STUDIES LINKING ACTIVATION OF MICROGLIAL AND PERIPHERAL MONOCYTIC CELLS TO PSYCHIATRIC DISORDERS}

Several animal models are characterized by abnormalities in both immune system function and behavior (Amrani et al., 1994; Yirmiya, 1996; Bothe et al., 2005; Frenois et al., 2007; Fu et al., 2010). These models have enabled us to observe the activation of circulating monocytes and microglia in the brain and to investigate their influence on behavior. The non-obese diabetic (NOD) mouse spontaneously develops autoimmune diabetes, which is similar to the onset of type 1 diabetes in humans. Psychiatric diseases in humans have been associated with autoimmune diseases, such as type 1 diabetes and autoimmune thyroiditis (Kupka et al., 2002; Padmos et al., 2004; Eaton et al., 2010). Interestingly, abnormal behaviors have also been observed in NOD mice (Amrani et al., 1994; Bothe et al., 2005). This model is useful for investigating associations between microglial activation and monocytes in pathological processes, as well as gene-environment interactions in this association. In addition, ion channels have recently been recognized to play important roles in the immune response of neurological disorders (Eder, 2010). Local changes in cell

\section{REFERENCES}

Amrani, A., Chaouloff, F., Mormede, P., Dardenne, M., and Homo-Delarche, F. (1994). Glucose, insulin and open field responses to immobilization in nonobese diabetic (NOD) mice. Physiol. Behav. 56, 241-246. doi: 10.1016/00319384(94)90190-2

Anisman, H. (2009). Cascading effects of stressors and inflammatory immune system activation: implications for major depressive disorder. J. Psychiatry Neurosci. 34, 4-20.

Banks, W. A., and Erickson, M. A. (2010). The blood-brain barrier and immune function and dysfunction. Neurobiol. Dis. 37, 26-32. doi: 10.1016/j.nbd.2009. 07.031

Banks, W. A., Gray, A. M., Erickson, M. A., Salameh, T. S., Damodarasamy, M., Sheibani, N., et al. (2015). Lipopolysaccharide-induced blood-brain barrier disruption: roles of cyclooxygenase, oxidative stress, neuroinflammation and osmolality enable monocytes to migrate and invade the CNS parenchyma, where they further differentiate into phagocytes under pathological conditions (Fuentes et al., 1995; Bennett et al., 2003; Mahad and Ransohoff, 2003; Schwab et al., 2007). Ion channels also have important roles in the process of microglia activation (Eder, 2010). Therefore, ion channel inhibitors are good candidates for therapeutic interventions to control immune responses in psychiatric diseases (Wulff et al., 2000; Reich et al., 2005; Rangaraju et al., 2009; Eder, 2010).

\section{CONCLUSION}

Microglia and monocytes have similar functions, surface markers, and gene expression profiles. Both cell types release pro-inflammatory cytokines when activated in response to stimuli in the brain under various pathological conditions. Accumulating data also indicate interactions between monocytes and microglia. Research into these interactions may lead to new strategies to elucidate the pathogenesis of psychiatric diseases, as well as to develop peripheral biomarkers that reflect the pathological conditions in the brain, including microglial activation related to the progression or modulation of disease.

\section{AUTHOR CONTRIBUTIONS}

HT contributed to the design of the manuscript. YT and HT drafted the manuscript. All authors contributed to construction of the context, revision of the manuscript. All authors read and approved the final manuscript.

\section{ACKNOWLEDGMENTS}

This work was supported by a grant-in-aid for scientific research on innovative areas (no. 24116007) from the Ministry of Education, Culture, Sports, Science, and Technology of Japan, Health and Labour Sciences Research Grants for research on psychiatric and neurological diseases and mental health (H19-kokoro-ippan-001), and an Intramural Research Grant (No. 21-9) for Neurological and Psychiatric Disorders from the National Center of Neurology and Psychiatry.

elements of the neurovascular unit. J. Neuroinflammation 12:223. doi: 10 1186/s12974-015-0434-1

Banks, W. A., and Robinson, S. M. (2010). Minimal penetration of lipopolysaccharide across the murine blood-brain barrier. Brain Behav. Immun. 24, 102-109. doi: 10.1016/j.bbi.2009.09.001

Barger, S. W., and Basile, A. S. (2001). Activation of microglia by secreted amyloid precursor protein evokes release of glutamate by cystine exchange and attenuates synaptic function. J. Neurochem. 76, 846-854. doi: 10.1046/j.14714159.2001.00075.x

Bayer, T. A., Buslei, R., Havas, L., and Falkai, P. (1999). Evidence for activation of microglia in patients with psychiatric illnesses. Neurosci. Lett. 271, 126-128. doi: 10.1016/s0304-3940(99)00545-5

Bennett, J. L., Elhofy, A., Dal Canto, M. C., Tani, M., Ransohoff, R. M., and Karpus, W. J. (2003). CCL2 transgene expression in the central nervous system directs diffuse infiltration of CD45highCD11b+ monocytes and 
enhanced Theiler's murine encephalomyelitis virus-induced demyelinating disease. J. Neurovirol. 9, 623-636. doi: 10.1080/714044484

Beumer, W., Gibney, S. M., Drexhage, R. C., Pont-Lezica, L., Doorduin, J., Klein, H. C., et al. (2012). The immune theory of psychiatric diseases: a key role for activated microglia and circulating monocytes. J. Leukoc. Biol. 92, 959-975. doi: $10.1189 / \mathrm{jlb} .0212100$

Boche, D., Perry, V. H., and Nicoll, J. A. (2013). Review: activation patterns of microglia and their identification in the human brain. Neuropathol. Appl. Neurobiol. 39, 3-18. doi: 10.1111/nan.12011

Bothe, G. W. M., Bolivar, V. J., Vedder, M. J., and Geistfeld, J. G. (2005). Behavioral differences among fourteen inbred mouse strains commonly used as disease models. Comp. Med. 55, 326-334.

Butovsky, O., Siddiqui, S., Gabriely, G., Lanser, A. J., Dake, B., Murugaiyan, G., et al. (2012). Modulating inflammatory monocytes with a unique microRNA gene signature ameliorates murine ALS. J. Clin. Invest. 122, 3063-3087. doi: 10. $1172 /$ jci62636

Calderó, J., Brunet, N., Ciutat, D., Hereu, M., and Esquerda, J. E. (2009). Development of microglia in the chick embryo spinal cord: implications in the regulation of motoneuronal survival and death. J. Neurosci. Res. 87, 2447-2466. doi: $10.1002 /$ jnr.22084

Chan, W. Y., Kohsaka, S., and Rezaie, P. (2007). The origin and cell lineage of microglia: new concepts. Brain Res. Rev. 53, 344-354. doi: 10.1016/j. brainresrev.2006.11.002

Chesnokova, V., and Melmed, S. (2002). Minireview: neuro-immuno-endocrine modulation of the hypothalamic-pituitary-adrenal (HPA) axis by gp130 signaling molecules. Endocrinology 143, 1571-1574. doi: 10.1210/en.143. 5.1571

Doorduin, J., de Vries, E. F., Willemsen, A. T., de Groot, J. C., Dierckx, R. A., and Klein, H. C. (2009). Neuroinflammation in schizophrenia-related psychosis: a PET study. J. Nucl. Med. 50, 1801-1807. doi: 10.2967/jnumed.109.066647

Drexhage, R. C., Hoogenboezem, T. H., Versnel, M. A., Berghout, A., Nolen, W. A., and Drexhage, H. A. (2011). The activation of monocyte and T cell networks in patients with bipolar disorder. Brain Behav. Immun. 25, 1206-1213. doi: 10. 1016/j.bbi.2011.03.013

Drexhage, R. C., Padmos, R. C., de Wit, H., Versnel, M. A., Hooijkaas, H., van der Lely, A.-J., et al. (2008). Patients with schizophrenia show raised serum levels of the pro-inflammatory chemokine CCL2: association with the metabolic syndrome in patients? Schizophr. Res. 102, 352-355. doi: 10.1016/j.schres.2008. 03.018

Drexhage, R. C., van der Heul-Nieuwenhuijsen, L., Padmos, R. C., van Beveren, N., Cohen, D., Versnel, M. A., et al. (2010). Inflammatory gene expression in monocytes of patients with schizophrenia: overlap and difference with bipolar disorder. A study in naturalistically treated patients. Int. J. Neuropsychopharmacol. 13, 1369-1381. doi: 10.1017/s1461145710000799

Eaton, W. W., Pedersen, M. G., Nielsen, P. R., and Mortensen, P. B. (2010). Autoimmune diseases, bipolar disorder and non-affective psychosis. Bipolar Disord. 12, 638-646. doi: 10.1111/j.1399-5618.2010.00853.x

Eder, C. (2010). Ion channels in monocytes and microglia/brain macrophages: promising therapeutic targets for neurological diseases. J. Neuroimmunol. 224, 51-55. doi: 10.1016/j.jneuroim.2010.05.008

Ekdahl, C. T. (2012). Microglial activation-tuning and pruning adult neurogenesis. Front. Pharmacol. 3:41. doi: 10.3389/fphar.2012.00041

Esposito, P., Gheorghe, D., Kandere, K., Pang, X., Connolly, R., Jacobson, S., et al. (2001). Acute stress increases permeability of the blood-brain-barrier through activation of brain mast cells. Brain Res. 888, 117-127. doi: 10.1016/s00068993(00)03026-2

Fillman, S. G., Cloonan, N., Catts, V. S., Miller, L. C., Wong, J., McCrossin, T., et al. (2013). Increased inflammatory markers identified in the dorsolateral prefrontal cortex of individuals with schizophrenia. Mol. Psychiatry 18, 206-214. doi: 10.1038/mp.2012.110

Fogg, D. K., Sibon, C., Miled, C., Jung, S., Aucouturier, P., Littman, D. R., et al. (2006). A clonogenic bone marrow progenitor specific for macrophages and dendritic cells. Science 311, 83-87. doi: 10.1126/science.1117729

Frenois, F., Moreau, M., O'Connor, J., Lawson, M., Micon, C., Lestage, J., et al. (2007). Lipopolysaccharide induces delayed FosB/DeltaFosB immunostaining within the mouse extended amygdala, hippocampus and hypothalamus, that parallel the expression of depressive-like behavior. Psychoneuroendocrinology 32, 516-531. doi: 10.1016/j.psyneuen.2007.03.005
Fu, X., Zunich, S. M., O'Connor, J. C., Kavelaars, A., Dantzer, R., and Kelley, K. W. (2010). Central administration of lipopolysaccharide induces depressive-like behavior in vivo and activates brain indoleamine 2,3 dioxygenase in murine organotypic hippocampal slice cultures. J. Neuroinflammation 7:43. doi: 10. 1186/1742-2094-7-43

Fuentes, M. E., Durham, S. K., Swerdel, M. R., Lewin, A. C., Barton, D. S., Megill, J. R., et al. (1995). Controlled recruitment of monocytes and macrophages to specific organs through transgenic expression of monocyte chemoattractant protein-1. J. Immunol. 155, 5769-5776.

Ginhoux, F., Greter, M., Leboeuf, M., Nandi, S., See, P., Gokhan, S., et al. (2010). Fate mapping analysis reveals that adult microglia derive from primitive macrophages. Science 330, 841-845. doi: 10.1126/science.1194637

Goldmann, T., Wieghofer, P., Muller, P. F., Wolf, Y., Varol, D., Yona, S., et al. (2013). A new type of microglia gene targeting shows TAK1 to be pivotal in CNS autoimmune inflammation. Nat. Neurosci. 16, 1618-1626. doi: 10. 1038/nn.3531

Gordon, S., and Taylor, P. R. (2005). Monocyte and macrophage heterogeneity. Nat. Rev. Immunol. 5, 953-964. doi: 10.1038/nri1733

Haarman, B. C., Riemersma-Van der Lek, R. F., de Groot, J. C., Ruhé, H. G., Klein, H. C., Zandstra, T. E., et al. (2014). Neuroinflammation in bipolar disorder - A [(11)C]-(R)-PK11195 positron emission tomography study. Brain Behav. Immun. 40, 219-225. doi: 10.1016/j.bbi.2014.03.016

Hayashi, M., Takai, J., Yu, L., Motohashi, H., Moriguchi, T., and Yamamoto, M. (2015). Whole-body in vivo monitoring of inflammatory diseases exploiting human interleukin 6-luciferase transgenic mice. Mol. Cell Biol. 35, 3590-3601. doi: 10.1128/MCB.00506-15

Hercher, C., Chopra, V., and Beasley, C. L. (2014). Evidence for morphological alterations in prefrontal white matter glia in schizophrenia and bipolar disorder. J. Psychiatry Neurosci. 39, 376-385. doi: 10.1503/jpn.130277

Hu, X., Leak, R. K., Shi, Y., Suenaga, J., Gao, Y., Zheng, P., et al. (2015). Microglial and macrophage polarization new prospects for brain repair. Nat. Rev. Neurol. 11, 56-64. doi: 10.1038/nrneurol.2014.207

Iliff, J. J., Goldman, S. A., and Nedergaard, M. (2015). Implications of the discovery of brain lymphatic pathways. Lancet Neurol. 14, 977-979. doi: 10.1016/s14744422(15)00221-5

Kastin, A. J., Pan, W., Maness, L. M., and Banks, W. A. (1999). Peptides crossing the blood-brain barrier: some unusual observations. Brain Res. 848, 96-100. doi: 10.1016/s0006-8993(99)01961-7

Kierdorf, K., Erny, D., Goldmann, T., Sander, V., Schulz, C., Perdiguero, E. G., et al. (2013). Microglia emerge from erythromyeloid precursors via Pu.1- and Irf8-dependent pathways. Nat. Neurosci. 16, 273-280. doi: 10.1038/nn.3318

Kim, Y. K., Kim, L., and Lee, M. S. (2000). Relationships between interleukins, neurotransmitters and psychopathology in drug-free male schizophrenics. Schizophr. Res. 44, 165-175. doi: 10.1016/s0920-9964(99)00171-1

Kupka, R. W., Nolen, W. A., Post, R. M., McElroy, S. L., Altshuler, L. L., Denicoff, K. D., et al. (2002). High rate of autoimmune thyroiditis in bipolar disorder: lack of association with lithium exposure. Biol. Psychiatry 51, 305-311. doi: 10.1016/s0006-3223(01)01217-3

Lassmann, H., Schmied, M., Vass, K., and Hickey, W. F. (1993). Bone marrow derived elements and resident microglia in brain inflammation. Glia 7, 19-24. doi: 10.1002/glia.440070106

Lawson, L. J., Perry, V. H., Dri, P., and Gordon, S. (1990). Heterogeneity in the distribution and morphology of microglia in the normal adult mouse brain. Neuroscience 39, 151-170. doi: 10.1016/0306-4522(90)90229-w

Louveau, A., Smirnov, I., Keyes, T. J., Eccles, J. D., Rouhani, S. J., Peske, J. D., et al. (2015). Structural and functional features of central nervous system lymphatic vessels. Nature 523, 337-341. doi: 10.1038/nature14432

Lu, B., Wang, C., Wang, M., Li, W., Chen, F., Tracey, K. J., et al. (2014). Molecular mechanism and therapeutic modulation of high mobility group box 1 release and action: an updated review. Expert Rev. Clin. Immunol. 10, 713-727. doi: 10. 1586/1744666X.2014.909730

Lutter, D., Ugocsai, P., Grandl, M., Orso, E., Theis, F., Lang, E. W., et al. (2008). Analyzing M-CSF dependent monocyte/macrophage differentiation: expression modes and meta-modes derived from an independent component analysis. BMC Bioinformatics 9:100. doi: 10.1186/1471-2105-9-100

Mahad, D. J., and Ransohoff, R. M. (2003). The role of MCP-1 (CCL2) and CCR2 in multiple sclerosis and experimental autoimmune encephalomyelitis (EAE). Semin. Immunol. 15, 23-32. doi: 10.1016/s1044-5323(02)00125-2 
Maiorino, C., Khorooshi, R., Ruffini, F., Løbner, M., Bergami, A., Garzetti, L., et al. (2013). Lentiviral-mediated administration of IL-25 in the CNS induces alternative activation of microglia. Gene Ther. 20, 487-496. doi: 10.1038/gt. 2012.58

Marshall, S. A., McClain, J. A., Kelso, M. L., Hopkins, D. M., Pauly, J. R., and Nixon, K. (2013). Microglial activation is not equivalent to neuroinflammation in alcohol-induced neurodegeneration: the importance of microglia phenotype. Neurobiol. Dis. 54, 239-251. doi: 10.1016/j.nbd.2012.12.016

Mignini, F., Streccioni, V., and Amenta, F. (2003). Autonomic innervation of immune organs and neuroimmune modulation. Auton. Autacoid Pharmacol. 23, 1-25. doi: 10.1046/j.1474-8673.2003.00280.x

Mildner, A., Schlevogt, B., Kierdorf, K., Böttcher, C., Erny, D., Kummer, M. P., et al. (2011). Distinct and non-redundant roles of microglia and myeloid subsets in mouse models of Alzheimer's disease. J. Neurosci. 31, 11159-11171. doi: 10.1523/JNEUROSCI.6209-10.2011

Naudin, J., Capo, C., Giusano, B., Mège, J., and Azorin, J. (1997). A differential role for interleukin-6 and tumor necrosis factor- $\alpha$ in schizophrenia? Schizophr. Res. 26, 227-233. doi: 10.1016/s0920-9964(97)00059-5

Nikkilä, H. V., Müller, K., Ahokas, A., Miettinen, K., Rimón, R., and Andersson, L. C. (2014). Accumulation of macrophages in the CSF of schizophrenic patients during acute psychotic episodes. Am. J. Psychiatry 156, 1725-1729.

Orihuela, R., McPherson, C. A., and Harry, G. J. (2016). Microglial M1/M2 polarization and metabolic states. Br. J. Pharmacol. 173, 649-665. doi: 10. 1111/bph.13139

Padmos, R. C., Bekris, L., Knijff, E. M., Tiemeier, H., Kupka, R. W., Cohen, D., et al. (2004). A high prevalence of organ-specific autoimmunity in patients with bipolar disorder. Biol. Psychiatry 56, 476-482. doi: 10.1016/j.biopsych.2004. 07.003

Padmos, R. C., Hillegers, M. H., Knijff, E. M., Vonk, R., Bouvy, A., Staal, F. J., et al. (2008). A discriminating messenger RNA signature for bipolar disorder formed by an aberrant expression of inflammatory genes in monocytes. Arch. Gen. Psychiatry 65, 395-407. doi: 10.1001/archpsyc.65.4.395

Papadopoulos, V., Baraldi, M., Guilarte, T. R., Knudsen, T. B., Lacapère, J. J., Lindemann, P., et al. (2006). Translocator protein (18kDa): new nomenclature for the peripheral-type benzodiazepine receptor based on its structure and molecular function. Trends Pharmacol. Sci. 27, 402-409. doi: 10.1016/j.tips. 2006.06.005

Parkhurst, C. N., Yang, G., Ninan, I., Savas, J. N., Yates, J. R. III, Lafaille, J. J., et al. (2013). Microglia promote learning-dependent synapse formation through brain-derived neurotrophic factor. Cell 155, 1596-1609. doi: 10.1016/j.cell. 2013.11.030

Prinz, M., and Priller, J. (2014). Microglia and brain macrophages in the molecular age: from origin to neuropsychiatric disease. Nat. Rev. Neurosci. 15, 300-312. doi: $10.1038 / \mathrm{nrn} 3722$

Radewicz, K., Garey, L. J., Gentleman, S. M., and Reynolds, R. (2000). Increase in HLA-DR immunoreactive microglia in frontal and temporal cortex of chronic schizophrenics. J. Neuropathol. Exp. Neurol. 59, 137-150. doi: 10.1093/jnen/59. 2.137

Rangaraju, S., Chi, V., Pennington, M. W., and Chandy, K. G. (2009). Kv1. 3 potassium channels as a therapeutic target in multiple sclerosis. Expert Opin. Ther. Targets 13, 909-924. doi: 10.1517/14728220903018957

Reich, E. P., Cui, L., Yang, L., Pugliese-Sivo, C., Golovko, A., Petro, M., et al. (2005). Blocking ion channel KCNN4 alleviates the symptoms of experimental autoimmune encephalomyelitis in mice. Eur. J. Immunol. 35, 1027-1036. doi: 10.1002/eji.200425954

Réus, G. Z., Fries, G. R., Stertz, L., Badawy, M., Passos, I. C., Barichello, T., et al. (2015). The role of inflammation and microglial activation in the pathophysiology of psychiatric disorders. Neuroscience 300, 141-154. doi: 10. 1016/j.neuroscience.2015.05.018

Rock, R. B., Gekker, G., Hu, S., Sheng, W. S., Cheeran, M., Lokensgard, J. R., et al. (2004). Role of microglia in central nervous system infections. Clin. Microbiol. Rev. 17, 942-964. doi: 10.1128/cmr.17.4.942-964.2004

Rothe, G., Herr, A. S., Stöhr, J., Abletshauser, C., Weidinger, G., and Schmitz, G. (1999). A more mature phenotype of blood mononuclear phagocytes is induced by fluvastatin treatment in hypercholesterolemic patients with coronary heart disease. Atherosclerosis 144, 251-261. doi: 10.1016/s0021-9150(99) 00061-1
Rothermundt, M., Arolt, V., Weitzsch, C., Eckhoff, D., and Kirchner, H. (1998). Immunological dysfunction in schizophrenia: a systematic approach. Neuropsychobiology 37, 186-193. doi: 10.1159/000026501

Schmitz, G., and Grandl, M. (2007). Role of redox regulation and lipid rafts in macrophages during Ox-LDL-mediated foam cell formation. Antioxid. Redox Signal. 9, 1499-1518. doi: 10.1089/ars.2007.1663

Schmitz, G., Leuthäuser-Jaschinski, K., and Orsó, E. (2009). Are circulating monocytes as microglia orthologues appropriate biomarker targets for neuronal diseases? Cent. Nerv. Syst. Agents Med. Chem. 9, 307-330. doi: 10. 2174/187152409789630424

Schmitz, G., Orsó, E., Rothe, G., and Klucken, J. (1997). Scavenging, signalling and adhesion coupling in macrophages: implications for atherogenesis. Curr. Opin. Lipidol. 8, 287-300. doi: 10.1097/00041433-199710000-00008

Schulz, C., Gomez Perdiguero, E., Chorro, L., Szabo-Rogers, H., Cagnard, N., Kierdorf, K., et al. (2012). A lineage of myeloid cells independent of Myb and hematopoietic stem cells. Science 336, 86-90. doi: 10.1126/science.1219179

Schwab, A., Nechyporuk-Zloy, V., Fabian, A., and Stock, C. (2007). Cells move when ions and water flow. Pflugers Arch. 453, 421-432. doi: 10.1007/s00424006-0138-6

Shechter, R., London, A., Varol, C., Raposo, C., Cusimano, M., Yovel, G., et al (2009). Infiltrating blood-derived macrophages are vital cells playing an antiinflammatory role in recovery from spinal cord injury in mice. PLoS Med. 6:e1000113. doi: 10.1371/journal.pmed.1000113

Shechter, R., and Schwartz, M. (2013). Harnessing monocyte-derived macrophages to control central nervous system pathologies: no longer 'if but 'how'. J. Pathol. 229, 332-346. doi: 10.1002/path.4106

Simard, A. R., Soulet, D., Gowing, G., Julien, J. P., and Rivest, S. (2006). Bone marrow-derived microglia play a critical role in restricting senile plaque formation in Alzheimer's disease. Neuron 49, 489-502. doi: 10.1016/j.neuron. 2006.01.022

Song, X. Q., Lv, L. X., Li, W. Q., Hao, Y. H., and Zhao, J. P. (2009). The interaction of nuclear factor-kappa B and cytokines is associated with schizophrenia. Biol. Psychiatry 65, 481-488. doi: 10.1016/j.biopsych.2008.10.018

Spiegel, A., Kalinkovich, A., Shivtiel, S., Kollet, O., and Lapidot, T. (2008). Stem cell regulation via dynamic interactions of the nervous and immune systems with the microenvironment. Cell Stem Cell 3, 484-492. doi: 10.1016/j.stem.2008. 10.006

Stamatovic, S., Dimitrijevic, O., Keep, R., and Andjelkovic, A. (2006). "Inflammation and brain edema: new insights into the role of chemokines and their receptors," in Brain Edema XIII, eds J. T. Hoff, R. F. Keep, G. Xi and Y. Hua (Vienna: Springer), 444-450.

Stöhr, J., Schindler, G., Rothe, G., and Schmitz, G. (1998). Enhanced upregulation of the fc $\gamma$ receptor IIIa (CD16a) during in vitro differentiation of ApoE4/4 monocytes. Arterioscler. Thromb. Vasc. Biol. 18, 1424-1432. doi: 10.1161/01. atv.18.9.1424

Streit, W. J., Walter, S. A., and Pennell, N. A. (1999). Reactive microgliosis. Prog. Neurobiol. 57, 563-581. doi: 10.1016/s0301-0082(98)00069-0

Takaki, J., Fujimori, K., Miura, M., Suzuki, T., Sekino, Y., and Sato, K. (2012). L-glutamate released from activated microglia downregulates astrocytic L-glutamate transporter expression in neuroinflammation: the 'collusion'hypothesis for increased extracellular L-glutamate concentration in neuroinflammation. J. Neuroinflammation 9:275. doi: 10.1186/17422094-9-275

Takano, A., Arakawa, R., Ito, H., Tateno, A., Takahashi, H., Matsumoto, R., et al. (2010). Peripheral benzodiazepine receptors in patients with chronic schizophrenia: a PET study with [11C]DAA1106. Int. J. Neuropsychopharmacol. 13, 943-950. doi: 10.1017/s1461145710000313

Tang, B., Capitao, C., Dean, B., and Thomas, E. A. (2012). Differential age- and disease-related effects on the expression of genes related to the arachidonic acid signaling pathway in schizophrenia. Psychiatry Res. 196, 201-206. doi: 10. 1016/j.psychres.2011.09.026

Theodoropoulou, S., Spanakos, G., Baxevanis, C. N., Economou, M., Gritzapis, A. D., Papamichail, M. P., et al. (2001). Cytokine serum levels, autologous mixed lymphocyte reaction and surface marker analysis in never medicated and chronically medicated schizophrenic patients. Schizophr. Res. 47, 13-25. doi: 10.1016/s0920-9964(00)00007-4

Thomas, D. M., Francescutti-Verbeem, D. M., and Kuhn, D. M. (2006). Gene expression profile of activated microglia under conditions associated 
with dopamine neuronal damage. FASEB J. 20, 515-517. doi: 10.1096/fj.054873fje

Torres-Platas, S. G., Cruceanu, C., Chen, G. G., Turecki, G., and Mechawar, N. (2014). Evidence for increased microglial priming and macrophage recruitment in the dorsal anterior cingulate white matter of depressed suicides. Brain Behav. Immun. 42, 50-59. doi: 10.1016/j.bbi.2014.05.007

van Berckel, B. N., Bossong, M. G., Boellaard, R., Kloet, R., Schuitemaker, A., Caspers, E., et al. (2008). Microglia activation in recent-onset schizophrenia: a quantitative (R)-[11 C] PK11195 positron emission tomography study. Biol. Psychiatry 64, 820-822. doi: 10.1016/j.biopsych.2008.04.025

Vaughan, D. W., and Peters, A. (1974). Neuroglial cells in the cerebral cortex of rats from young adulthood to old age: an electron microscope study. J. Neurocytol. 3, 405-429. doi: 10.1007/bf01098730

Veenman, L., and Gavish, M. (2000). Peripheral-type benzodiazepine receptors: their implication in brain disease. Drug Dev. Res. 50, 355-370. doi: 10. 1002/1098-2299(200007/08)50:3/4<355::AID-DDR18>3.0.CO;2-W

Veenman, L., and Gavish, M. (2012). The role of $18 \mathrm{kDa}$ mitochondrial translocator protein (TSPO) in programmed cell death and effects of steroids on TSPO expression. Curr. Mol. Med. 12, 398-412. doi: 10. 2174/1566524011207040398

Whitelaw, D. M. (1972). Observations on human monocyte kinetics after pulse labeling. Cell Tissue Kinet. 5, 311-317. doi: 10.1111/j.1365-2184.1972. tb00369.x

Wiersinga, W. J., Leopold, S. J., Cranendonk, D. R., and van der Poll, T. (2014). Host innate immune responses to sepsis. Virulence 5, 36-44. doi: 10.4161/viru. 25436

Wulff, H., Miller, M. J., Hänsel, W., Grissmer, S., Cahalan, M. D., and Chandy, K. G. (2000). Design of a potent and selective inhibitor of the intermediate-conductance $\mathrm{Ca}^{2+}$-activated $\mathrm{K}^{+}$channel, IKCal: a potential immunosuppressant. Proc. Natl. Acad. Sci. U S A 97, 8151-8156. doi: 10. 1073/pnas.97.14.8151

Yirmiya, R. (1996). Endotoxin produces a depressive-like episode in rats. Brain Res. 711, 163-174. doi: 10.1016/0006-8993(95)01415-2

Yona, S., Kim, K. W., Wolf, Y., Mildner, A., Varol, D., Breker, M., et al. (2013). Fate mapping reveals origins and dynamics of monocytes and tissue macrophages under homeostasis. Immunity 38, 79-91. doi: 10.1016/j.immuni.2012. 12.001

Yu, Z., Ono, C., Aiba, S., Kikuchi, Y., Sora, I., Matsuoka, H., et al. (2015). Therapeutic concentration of lithium stimulates complement C3 production in dendritic cells and microglia via GSK-3 inhibition. Glia 63, 257-270. doi: 10. 1002/glia.22749

Conflict of Interest Statement: The authors declare that the research was conducted in the absence of any commercial or financial relationships that could be construed as a potential conflict of interest.

The reviewer MO and handling Editor declared their shared affiliation, and the handling Editor states that the process nevertheless met the standards of a fair and objective review.

Copyright (c) 2016 Takahashi, Yu, Sakai and Tomita. This is an open-access article distributed under the terms of the Creative Commons Attribution License (CC BY). The use, distribution and reproduction in other forums is permitted, provided the original author(s) or licensor are credited and that the original publication in this journal is cited, in accordance with accepted academic practice. No use, distribution or reproduction is permitted which does not comply with these terms. 